\title{
Surgimiento de las prácticas científicas de colaboración en la ciencia mexicana con cobertura en los índices internacionales
}

\author{
Francisco Collazo Reyes - Centro de Investigación y de Estudios Avanzados, \\ Departamento de Física, México ${ }^{1}$ \\ María Elena Luna Morales - Centro de Investigación y de Estudios Avanzados, \\ Coordinación General de Servicios Bibliográficos, México \\ Gabriel Vélez Cuartas - Universidad de Antioquia, Departamento de Sociología, \\ Colombia
}

\section{Resumen}

Presentamos un estudio de la producción científica mexicana de corriente principal publicada en la primera mitad del siglo XX, a través de un enfoque metodológico que combina elementos historiográficos, sociales y bibliométricos orientados a identificar y caracterizar la evolución de sus prácticas científicas. Artículos del Science Citation Index y SCOPUS son analizados a través de herramientas provenientes del análisis de redes sociales revelando las relaciones de co-autoría. EI análisis y la contrastación entre los hechos históricos de organización y las medidas de cohesión, centralidad y densidad de las estructuras de la red bibliométrica, ofrecen una abundante documentación referente a un proceso de enriquecimiento y diversificación de las formas de organización de la ciencia mexicana que da cuenta de una etapa de consolidación ocurrida principalmente en el periodo de 1930 a 1950.

Palabras clave: Historiografía de la ciencia mexicana, Análisis de redes bibliométricas.

\begin{abstract}
A study of mainstream Mexican scientific production from 1900 to 1950 is presented. A methodological mixture is made with historiographical and bibliometrical network analysis elements. This is presented in order to characterize the evolution of scientific practices. Articles are taken from Science Citation Index and SCOPUS and they are analized through co-authorship networks. Analysis and contrast between historical facts of scientific organization and cohesion, centrality and density measures of co-authorship networks show an enrichment and diversification of Mexican science organization. This demonstrates a consolidation stage between 1930 and 1950 in this country.
\end{abstract}

Key words: Historiography of Mexican science - Bibliometric network analysis.

\footnotetext{
${ }^{1}$ Enviar correspondencia a: fcollazo@fis.cinvestav.mx
} 


\section{I ntroducción}

Se presenta un estudio exploratorio ${ }^{2}$ de la producción científica mexicana difundida en revistas clasificadas como de corriente principal por su cobertura en el Science Citation Index, publicada en la primera mitad del siglo XX, con el objeto de identificar y caracterizar la evolución de sus prácticas científicas. Entendidas como un proceso historiográfico social de construcción de las condiciones locales (Suárez, 2005; Martínez, 2003), para el desarrollo de espacios promotores de una actividad científica más estructurada en sus formas de organización, producción y apropiación de los paradigmas científicos internacionales. En este sentido, el estudio se pregunta por el desarrollo institucional de la ciencia y sus condiciones de producción. De esta manera el calificar la evolución tendrá que ver directamente con las condiciones y no con los aportes realizados a cada uno de los campos especializados.

Si entendemos a la ciencia como una forma de racionalidad específica, distinta a otras formas de racionalidad en lo social. Es decir, la ciencia como un sistema social entre otros sistemas sociales, el enriquecimiento y la diversificación de sus prácticas de producción darán cuenta de la consolidación creciente de esta forma de racionalidad específica (Luhmann, 1996, 2007). Una de las formas constitutivas de la construcción científica es su continuidad y reproducción a través de la formación de grupos y comunidades ${ }^{3}$, que generalmente repercute en el incremento de la producción científica, como un importante indicador de consolidación, no solo en términos cuantitativos, sino en sus formas organizativas. Esto implica que a mayor consolidación mayores grados de estandarización en las prácticas y metodologías constructivas de investigación y generación de productos. Esta estandarización permite de alguna u otra forma, generar una mayor masa crítica, con mayores posibilidades de comunicación entre pares y colegas.

La consolidación de comunidades académicas en términos evolutivos es observada en este estudio a partir de dos enfoques metodológicos: uno historiográfico-social,

\footnotetext{
2 Se plantea como estudio exploratorio dentro de un programa mayor acerca de la descripción evolutiva de la ciencia mexicana en la primera mitad del siglo XX. Este trabajo forma parte de una serie de trabajos que se han publicado en otras revistas como el citado en este artículo realizado por LunaMorales, ME; Collazo-Reyes, F and Russell, J, 2007.

3 adquieren sentido gracias a la especialización funcional de la sociedad que ha especializado a su vez el lenguaje. En este caso, se habla del sistema científico como una gran cantidad de formas comunicativas disponibles que se hacen científicas por la disposición de sus contenidos (Luhmann, 1996). De esta forma, el sistema científico crea mecanismos o patrones de interacción disponibles entre personas, las cuales para permitir que la comunicación continúe se agrupan y forman comunidades que recrean las formas de comunicación científicas disponibles (Leydesdorff, 2001).
} 
que a través de datos históricos intenta reconstruir algunos de los eventos más importantes en la evolución de la ciencia mexicana de la primera mitad del siglo XX. Este se expande mediante el análisis de redes aplicado a la Bibliometría, como herramienta de investigación que permite generar medidas topológicas o, relaciones entre autores, por medio de la producción bibliográfica. Pero no sólo pone en evidencia las relaciones entre autores sino las estructuras que se conforman a través de las relaciones de trabajo conjunto (Leydesdorff, 2008; Yoshikane F...et al, 2009; Velden, Haque, Lagoze, 2010). Estas estructuras pueden significar varias cosas: configuraciones de campos disciplinares, científicos o, temáticas, conformación de centros-periferias en la producción bibliográfica, e inclusive la aparición de diferentes formas de colaboración dentro de la ciencia, como pretende demostrar este trabajo.

La bibliometría ha utilizado instrumentos estadísticos que le permiten observar autores relevantes, campos de mayor producción y países que concentran el desarrollo de ciertas ciencias (Delgado, Torres, Jiménez y Ruíz, 2006). El análisis de redes aplicado a la bibliometría permite observar de manera descriptiva y analítica la conformación de estructuras, profundizando en asuntos como el peso de ciertos autores a través de sus interrelaciones, el dominio o alcances de estos autores a partir de las relaciones de sus compañeros de trabajo o, co-autoría, así como otra gran cantidad de aspectos que pueden ser complementarios y más amplios que los análisis tradicionales (Pontille, 2003a, 2004). De esta manera es posible por medio del análisis estructural de las co-autorías de los artículos científicos de la primera mitad del siglo XX en México, establecer la aparición de nuevas formas de construcción de la ciencia nacional Mexicana.

En el intento de condensar estos planteamientos sociológicos, metodológicos y bibliométricos, Loet Leydesdorff $(2001,2008)$ en su propuesta cienciométrica propone entender el análisis bibliométrico de citaciones, en dos dimensiones originadas por el tipo de observación que se haga de los datos: los lazos que relacionan textos, los cuales permiten observaciones acerca de la evolución de los discursos científicos, y por tanto la evolución cognitiva de la ciencia; y los lazos existentes entre autores, los que expresan las relaciones que establece un autor con otro en su desempeño laboral, generando una especie de sistema de reconocimiento y recompensas mutuas al citar a otro de sus colegas (Leydesdorff, 1990). Esto nos permite observar lo cognitivo y lo social desde el análisis bibliométrico, utilizando los planteamientos de las redes sociales para mejorar la construcción de mapas de campos científicos en los que se puedan observar problematizaciones y respuestas a través de la evolución de teorías y metodologías. 
Este estudio se inclina más por el análisis de los lazos establecidos entre autores y el impacto en la producción científica y sus formas de organización.

Teniendo en cuenta estos planteamientos, este artículo parte de la hipótesis de que en México, el desarrollo de las disciplinas más tradicionales del sistema científico: medicina general, clínica y experimental, biología, ingenierías, física, química y biomedicina; consolidaron sus prácticas y tradiciones científicas en la primera mitad del siglo XX, con la aparición de formas organizativas más complejas y distintas de producción y colaboración científicas, que ocurrieron de forma paralela pero con procesos crecientes de complejización, con diferencias importantes dependiendo del campo científico temático y sus respectivas relaciones con su entorno. Esta consolidación se gestó en el proceso de diversificación del modo de producción científica tradicional basado en la autoría individual y caracterizada como una práctica científica poco desarrollada en estado amateur (Saldaña, 1994; Luna, Collazo y Russell, 2007) y complementada con la aparición de estilos de investigación más productivos y más dependientes del marco normativo de la ciencia internacional, que incluye entre sus valores principales la promoción de la colaboración científica, que históricamente ha representado un proceso de enriquecimiento de los límites de crecimiento de la ciencia con respecto a las prácticas científicas aisladas dependientes del trabajo individual (Ziman, 1994).

En este ejercicio utilizamos como fuentes de información base, dos sistemas bibliográficos multidisciplinarios, con estructuras de información estandarizada para aplicaciones bibliométricas: Science Citation Index $(\mathrm{SCl})$ y SCOPUS. Éstas nos permitieron identificar y recuperar la versión más completa de la producción científica mexicana, escrita en revistas de corriente principal con adscripción a instituciones localizadas en el país, durante el periodo 1900-1950. Aclaramos que somos conscientes de que los trabajos considerados de corriente principal y las fuentes utilizadas, no representan la totalidad de la producción científica generada en México en este periodo. En este sentido, la validez de los resultados reportados en este trabajo está dada por dos hechos: uno, la publicación científica es la única forma actual disponible que permite observar formas de relación en la aparición de co-autorías, por tanto son los datos o hechos disponibles que permiten dar cuenta de un fenómeno emergente en la ciencia (Pontille, 2003b). Dos, la forma actual de visualización de la ciencia es a través de estas grandes bases de datos y sus prestigios, así como las formas organizativas visibles para la sociedad pueden ser observadas a través de estas bases. Esto definitivamente tiene efectos importantes no sólo en las formas de relatar la organización misma de la ciencia, sino también, en la asignación de recursos para financiar investigaciones, reconocimientos y el 
establecimiento de juicios para la recomendación o no de políticas públicas de organismos como la OCDE y otras instancias multilaterales que impactan de alguna u otra forma la ciencia.

\section{Metodología}

\section{Recuperación y organización de la información}

Recuperamos 280 trabajos del periodo 1900-1950: 103 en SCl, 71 en SCOPUS, en ambos casos utilizamos la estrategia de búsqueda "México not New Mexico" en el campo "Address", y 106 a través de búsquedas individuales por autores de la ciencia mexicana identificados en distintas fuentes (De Gortari, 1963; Robles Glenn, 1971; Pérez Tamayo, 2005; Saldaña, 2005; Luna Morales, et al, 2007, 2009). Esta última tarea incluye la verificación de las direcciones de adscripción de los autores, ausentes en el $\mathrm{SCl}$, directamente en las fuentes de publicación, consultadas en los acervos impresos o electrónicos de la Universidad Nacional Autónoma de México y el Centro de Investigación y de Estudios Avanzados. De esta manera, los 280 trabajos recuperados cumplen con el criterio de estar publicados en revistas incluidas en $\mathrm{SCl}$.

Los registros bibliográficos recuperados se ajustaron a una misma estructura de datos. Se organizaron en tablas relacionales y campos bibliográficos administrados en una base de datos, que fue utilizada para el proceso de desagregación y normalización de los nombres de autores.

\section{Desarrollo de la red de relaciones de coautoría}

Identificamos un total de 229 autores únicos, que ligados al año de publicación de sus trabajos generaron una matriz de autoría cronológica de 346 autores-año, donde los autores aparecen por cada año de publicación de sus trabajos. Esta matriz fue procesada en Pajek (Nooy, Mrvar and Batagelj, 2005) como la información principal que dio origen a la red general de relaciones de coautoría de la ciencia mexicana de la primera mitad del siglo XX.

\section{Estructuras de análisis de la red bibliométrica}

Para efectos de estudio de la red anterior, se utilizaron diferentes estructuras de análisis. Por un lado se utilizaron dos particiones: la primera que agrupa los 280 trabajos en siete áreas temáticas generales, de acuerdo con las categorías 
asignadas a los trabajos en el $\mathrm{SCl}$ : (1) Biomedicina, (2) Física, (3) Química, (4) Biología, (5) Geofísica, (6) Ingenierías, (7) Medicina; y la segunda que los divide en forma cronológica en cinco décadas: (a) 1900-1910; (b) 1911-1920; (c) 19211930; (d) 1931-1940; (e) 1941-1950. Por otro lado, se utilizó como vector de la red la producción individual de autores por año de publicación.

\section{Análisis de la red bibliométrica}

Para desarrollar el análisis exploratorio, se realizó una descripción morfológica a través del análisis de las estructuras de la red bibliométrica de coautoría con el software Pajek y el uso de las siguientes operaciones principales: (1) detección de componentes y distinción de grupos por particiones referentes a la pertenencia de campos de especialización específicos, (2) centralidades en co-autorías y densidad de los campos específicos, (3) K-núcleos o subgrupos dentro de la red por densidad de relaciones mutuas de co-autorías, (4) análisis del comportamiento de las coautorías en cada campo científico a partir de la división por décadas de la primera mitad del siglo XX, (5) análisis interpretativo de correspondencia entre hechos históricos y de organización histórica de la ciencia y estructuras de la red.

\section{Análisis historiográfico}

En los anexos 6 y 7 se recuperaron datos historiográficos que plantean hechos importantes que permiten validar algunas de las conclusiones del estudio emanadas del apartado analítico empleando el análisis de redes bibliométricas.

Historiografía de la ciencia mexicana

Una parte importante de los pilares que sostienen la estructura organizacional de la ciencia mexicana moderna, se construyeron en las décadas de los años 30 y 40, principalmente durante los gobiernos de Lázaro Cárdenas del Río (1934-1940) y Manuel Avila Camacho (1940-1946). Se crearon diferentes instituciones y dependencias relevantes en los distintos sectores de salud, gubernamental, industrial y de educación superior e investigación; comprometidas con objetivos de enseñanza y construcción de espacios para el desarrollo de prácticas de investigación, como se muestra en la tabla 6 (Anexos). Se fundaron entidades con objetivos orientados a la promoción de la ciencia y tecnología, a través de programas de financiamiento a proyectos y formación de recursos humanos. Las 
comunidades académicas y de investigación intensificaron los mecanismos de interacción entre colegas, a través de la formación de academias, asociaciones y sociedades científicas, como espacios tradicionales para la promoción de la discusión académica, la comunicación personal y el establecimiento de relaciones de colaboración científica, tabla 7 (Anexos).

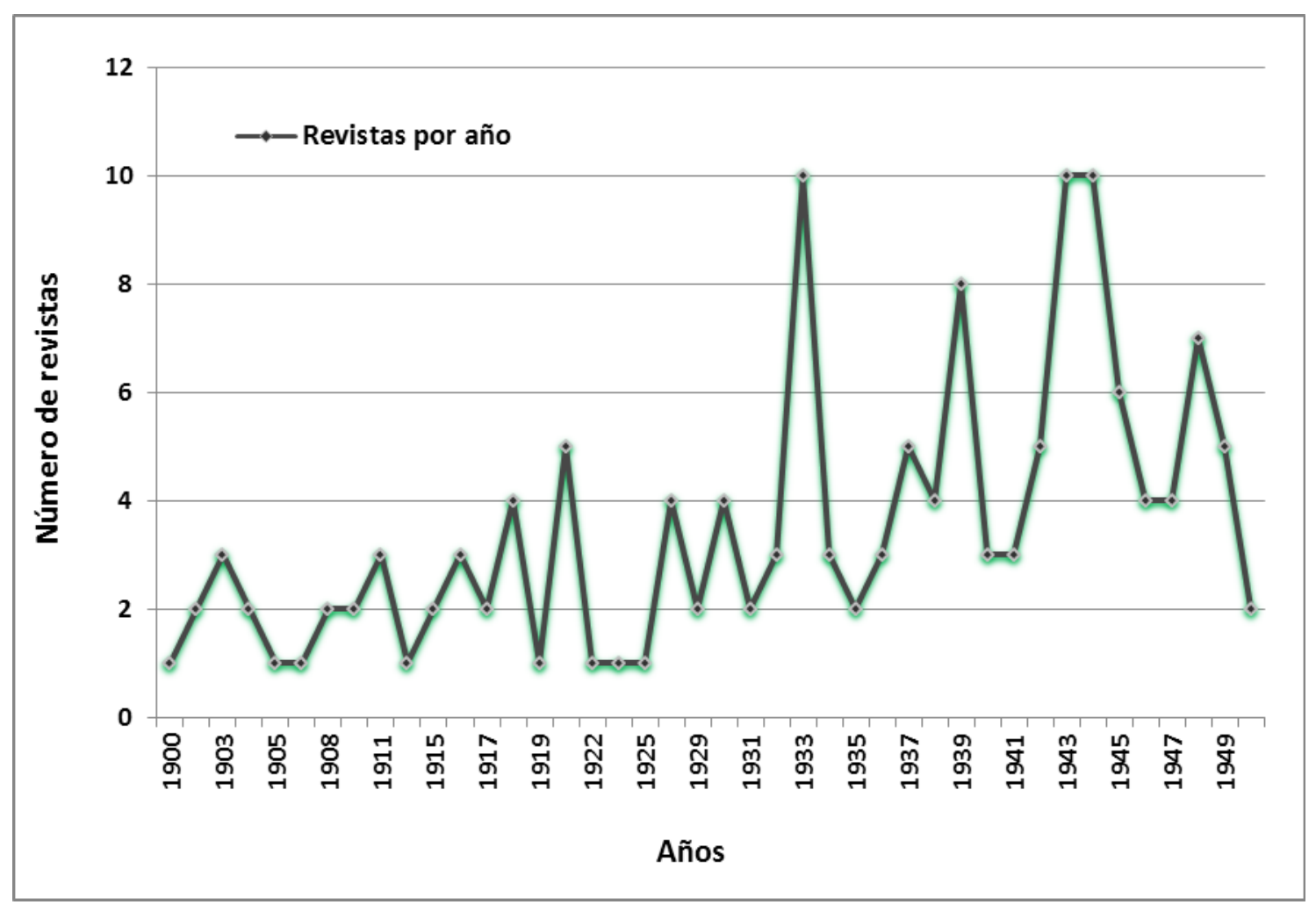

Figura 1. Número de nuevos títulos de revistas creados por año: 1900-1950.

En este periodo también surgieron el mayor número de títulos de revistas, de contenidos científicos, editadas durante la primera mitad del siglo XX, como se muestra en la figura 1. Revistas de divulgación, de difusión de conocimientos científicos y de intercambio de conocimientos. Las revistas son editadas principalmente por instituciones de investigación del área de salud, del sector académico e investigación y por asociaciones científicas; entre las que se encuentran la Revista de Investigación Clínica, Archivos del Instituto de Cardiología, Ciencia, Anales del Instituto de Biología, Boletín del Instituto de Química y el Boletín de la Sociedad Matemática Mexicana. Estos títulos fueron los primeros casos de publicaciones mexicanas en ser consideradas en la cobertura del Science Citation Index.

También se aplicaron políticas favorables a la formación de recursos humanos con grado académico de doctor, de atracción de investigadores externos interesados en 
el estudio de fenómenos de interés científico locales, y de apertura al desarrollo y participación de las comunidades científicas locales en congresos internacionales, a nivel local e internacional.

La preocupación del Gobierno Federal por los problemas de salud e higiene públicas y por hacer de México un lugar libre de enfermedades contagiosas y seguro para la atracción de inversiones, están presentes en la gran cantidad de recursos invertidos en la creación de los institutos de investigación en salud, mencionados en la tabla 7 (Anexos), En términos generales las ciencias de la salud fueron los campos con mayor apoyo del Gobierno Federal, en su propósito de generar políticas para la salud pública.

En el ámbito universitario se crearon universidades públicas, estatales, privadas, institutos tecnológicos y entidades dedicadas a la investigación. Bajo esta estructura de organización se desarrolló el proceso de conformación del sector de la educación superior y la matriz institucional responsable de la producción de conocimientos en el sector académico, durante el siglo XX. La investigación universitaria se inició en los institutos académicos de la UNAM, las escuelas superiores del IPN, las Universidades estatales y las privadas, mencionadas en la tabla 6 (Anexos).

A finales de la década de los años 30 se creó el Instituto de Física en la UNAM y se contrataron los primeros investigadores con grado de doctor en el área. (tabla 6). Aquí surgió el primer tema de la ciencia moderna con sede en espacios académicos (Collazo, Herrera, 2008), no necesariamente relacionado con problemas locales, aspecto que puso en práctica la libertad académica que le confiere la autonomía universitaria otorgada en 1929.

La biología se desarrolló fuertemente ligada a las actividades de enseñanza de botánica, zoología y a una enorme tradición descriptiva de la flora y la fauna de México (Ledesma; Barahona, 1999). Actividades que se complementaban con la promoción de expediciones que permitían continuar con el reconocimiento, la clasificación y el desarrollo de taxonomías y mapas de los recursos naturales biológicos y los ambientes climáticos de su desarrollo (Valiente, Flores, Verdú y Dávila, 1998).

A diferencia de las áreas de medicina, biomedicina y física, dependientes del financimiento público y de lentos procesos de construcción de instalaciones y de incorporación de recursos humanos especializados. Las características de la producción de conocimientos en el sector industrial presentan una organización distinta. Se contrató un grupo de prestigiados científicos para competir a nivel 
internacional en la obtención de resultados de investigación sobre los aspectos químicofarmacológicos de los esteroides. Se crearon instalaciones especializadas para el desarrollo de la investigación bajo el nombre comercial de Laboratorios Syntex. Los proyectos más relevantes, fueron financiados por empresas multinacionales interesadas en el desarrollo de esteroides $y$ agentes anticonceptivos. El interés de estas empresas estaba orientado a generar productos innovativos y competentes en el mercado, para ello conformaron un grupo de investigación corporativa, altamente cohesionado, y ligado a las principales corrientes científicas del área y a sus formas de institucionalización. De allí el fortalecimiento de la producción colaborativa.

La investigación en geociencias, ingeniería civil y metalurgia es escasa y se publicó principalmente durante las cuatro primeras décadas: 1900-1940. Fue desarrollada principalmente por investigadores con perfil profesional de ingenieros. Las publicaciones en las ingenierías y la metalurgia estuvieron financiadas principalmente por fondos de empresas transnacionales instaladas en México y en menor medida por instituciones del Gobierno Federal. Se desarrolló con fines utilitarios y en aspectos prácticos de intereses particulares, orientados a encontrar mejoras en los procedimientos de explotación y extracción de los recursos minerales y el petróleo. La disminución de este tipo de investigaciones coincide con el periodo de expropiación de la industria del petróleo en México y la expulsión de las empresas extranjeras dedicadas a su explotación.

\section{Resultados: morfología de la estructuras de colaboración en las disciplinas de la primera mitad del siglo $\mathbf{X X}$}

\section{Componentes de colaboración}

La tabla 1 muestra la estructura de colaboración en los textos por cada una de las disciplinas. Medicina general es la disciplina con una mayor estructura colaborativa, contrastan sus 122 autores con Biomedicina, Física y Química, que tienen 51, 47 y 54 investigadores, más del doble de cada una. Esto influye en la producción colaborativa de la ciencia desarrollada a finales de la primera mitad del siglo XX. Lo que significa una alta institucionalización de la medicina general. 


\begin{tabular}{|c|l|c|c|c|c|c|}
\hline No. & Disciplina & $\begin{array}{c}\text { Autores } \\
\text { por } \\
\text { disciplina }\end{array}$ & $\begin{array}{c}\text { Componentes } \\
\text { de } \\
\text { colaboración }\end{array}$ & $\begin{array}{c}\text { No. máximo } \\
\text { de } \\
\text { componentes }\end{array}$ & $\begin{array}{c}\text { No. mínimo } \\
\text { de } \\
\text { componentes }\end{array}$ & $\begin{array}{c}\text { Un } \\
\text { solo autor }\end{array}$ \\
\hline 1 & Biomedicina & 51 & 13 & 6 & 2 & 8 \\
\hline 2 & Física & 47 & 9 & 4 & 2 & 19 \\
\hline 3 & Química & 54 & 8 & 13 & 2 & 15 \\
\hline 4 & Biología & 26 & 5 & 5 & 2 & 12 \\
\hline 5 & Geociencias & 26 & 1 & 3 & - & 23 \\
\hline 6 & Ingenierías & 20 & 3 & 2 & - & 14 \\
\hline 7 & Medicina & 122 & 30 & 6 & 2 & 27 \\
\hline
\end{tabular}

Tabla 1. Estructuras de colaboración por disciplina en la ciencia mexicana.

Un componente de colaboración, se refiere a una subred dentro de la red donde cada vértice está conectado con el resto de vértices por lo menos a través de un camino. En este contexto, una subred se referiría a relaciones de colaboración de la producción de literatura científica donde cada autor que forma parte de un componente por lo menos ha realizado una obra con alguno de los integrantes.

Los 4 campos más relevantes en estructuras colaborativas, fueron también los más productivos en términos del número de trabajos generados: la medicina general, la biomedicina, la física y la química.

Con respecto a la colaboración como característica destacada por la convergencia de gran cantidad de autores en obras individuales, la química es la que mayor cantidad de autores aporta a los componentes de colaboración. En esta disciplina se pueden encontrar componentes colaborativos hasta con 13 autores. También presenta patrones de concurrencia repetitiva entre autores, por ejemplo aparecen dos autores con 10 colaboraciones distintas, diferente a los otros campos en donde no se encuentra producción con estas características. Esto da idea de un alto desarrollo organizativo y la existencia de programas de investigación con continuidad en términos colaborativos.

De igual manera destacan los campos de la medicina y la biomedicina, corroborando la colaboración como una forma institucional relevante permitiendo un desarrollo más armónico y comunicado. Esta también es una característica esencial de la ciencia, que solo puede darse si hay comunidades colaborativas que permiten dispersión y transferencia del conocimiento.

\section{Cantidad de relaciones de colaboración}

La cantidad de relaciones de colaboración entre dos o más autores, da cuenta de grados de consolidación en la organización de la ciencia. Las colaboraciones reiteradas, pueden denotar la continuidad en programas de investigación y el afán 
por hacer comunicable el trabajo conjunto, lo cual a su vez connota posibles comunidades científicas emergentes, en consolidación o consolidadas.

\begin{tabular}{|c|c|l|c|}
\hline Clasificación & Publicaciones & \multicolumn{1}{|c|}{ Autores Participantes } & Año \\
\hline 1 & 10 & López, J y Marker, RE, - & 1947 \\
\hline 2 & 5 & Djerassi, C y Rosenkranz, G & 1950 \\
\hline 3 & 3 & Gay, MAP y Varela, G & 1934 \\
\hline 4 & 3 & Djerassi, C y Kaufmann, ST & 1950 \\
\hline 5 & 3 & Djerassi, C y Pataki, J & 1950 \\
\hline 6 & 3 & Djerassi, C y Rosenkranz, G & 1950 \\
\hline 7 & 3 & Kaufmann, ST y Pataki, J & 1950 \\
\hline 8 & 3 & Kaufmann, ST y Rosenkranz, G & 1950 \\
\hline 9 & 3 & Pataki, J y Rosenkranz, G & 1950 \\
\hline 10 & 2 & Castañeda, MR y Silva, R & 1944 \\
\hline 11 & 2 & Ramos, J y Rosenblueth, A, & 1949 \\
\hline 12 & 2 & Djerassi, C y Kaufmann, ST & 1950 \\
\hline 13 & 2 & Djerassi, C y Pataki, J & 1950 \\
\hline 14 & 2 & Djerassi, C y Romo, J & 1950 \\
\hline 15 & 2 & Djerassi, C y Romo, J & 1950 \\
\hline 16 & 2 & Djerassi, C y Rosenkranz, G & 1950 \\
\hline 17 & 2 & Djerassi, C y Scholz, CR & 1950 \\
\hline 18 & 2 & Gelin, G y Gómez, F & 1950 \\
\hline 19 & 2 & Kaufmann, ST y Pataki, J & 1950 \\
\hline 20 & 2 & Kaufmann, ST y Pataki, J & 1950 \\
\hline 21 & 2 & Kaufmann, ST y Romo, J & 1950 \\
\hline 22 & 2 & Kaufmann, ST y Rosenkranz, G & 1950 \\
\hline 23 & 2 & Romo, J y Rosenkranz, G & 1950 \\
\hline 24 & 2 & Romo, J y Rosenkranz, G & 1950 \\
\hline
\end{tabular}

Tabla 2. Autores con mayor número de colaboraciones por año.

La columna de clasificación se refiere a un orden descendente de acuerdo con el número de colaboraciones entre dos autores en un mismo año; la columna publicaciones corresponde al número de colaboraciones hechas en co-autoría entre dos autores; la columna autores hace referencia a los autores mencionados; la última columna es el año de la colaboración. La tabla fue realizada con el software Pajek. (De Nooy, Mrvar, and Batagelj, 2005)

Los químicos registran mayor productividad en un mismo año y mayor cantidad de colaboraciones reiterativas entre autores. La mayoría de las relaciones clasificadas en la tabla 2 pertenecen al campo de la química, excepto las referentes a los números 3, 10 y 11 que pertenecen a medicina y la 18 que corresponde a biomedicina.

Es curioso que a pesar del tamaño de la medicina general, que duplica y hasta sextuplica algunos otros campos en el número de autores, no se encuentren 
relaciones reiterativas. Esto da lugar a varias posibilidades: escasez de motivos entre miembros del campo para crear y preservar relaciones de coautoría, presencia de programas de investigación emergentes, una mayor cantidad de recursos para la investigación en el área, y la fragmentación del campo investigativo; en contraste con la química.

\section{Centralidad de los autores en su producción anual por especialidad científica}

La centralidad en relaciones de colaboración, se refiere a la cantidad de relaciones que cada autor sostiene con otros para colaborar en la producción de un artículo científico (definición de centralidad de grado ver De Nooy, Mrvar and Batagelj, 2005). Como se muestra en la tabla 3, mediante las columnas de: biomedicina, física, química, biología, geociencia, ingeniería civil y metalurgia, y medicina general; donde los encabezados de las filas se refieren a la cantidad de relaciones que tiene un autor por año. Los números contenidos en la tabla se refieren a la cantidad de autores que tienen determinado número de colaboraciones (filas) en una determinada ciencia (columnas). La tabla ha sido realizada con el algoritmo Cramer's V, Rajski el cual reporta una tabulación cruzada y correlaciona dos propiedades en una estructura en red y ha sido realizada con Pajek (De Nooy, Mrvar and Batagelj, 2005).

La química es la que tiene más relaciones de un autor por año con otros autores, donde se observa alta productividad de unos pocos a través de la colaboración. A diferencia de medicina y biomedicina que no tienen autores por año con alta centralidad en colaboración, pero sí tienen pequeños proyectos colaborativos en mayor cantidad. La otra especialidad destacada, es la física, pero en menor grado que las tres disciplinas anteriores en centralidad de colaboración entre sus científicos. Ingeniería civil, metalurgia y geofísica tienen muy bajos niveles de colaboración entre sus científicos. 


\begin{tabular}{|c|c|c|c|c|c|c|c|c|}
\hline No. & \multicolumn{9}{|c|}{ Áreas temáticas } & Total \\
\cline { 1 - 8 } Relaciones & Biomedicina & Física & Química & Biología & Geociencias & Ingenierías & Medicina & \\
\hline 0 & 8 & 19 & 15 & 12 & 23 & 14 & 27 & 118 \\
\hline 1 & 17 & 8 & 9 & 6 & 0 & 6 & 27 & 73 \\
\hline 2 & 12 & 10 & 9 & 0 & 3 & 0 & 37 & 71 \\
\hline 3 & 12 & 10 & 5 & 2 & 0 & 0 & 19 & 48 \\
\hline 4 & 1 & 0 & 2 & 6 & 0 & 0 & 12 & 21 \\
\hline 5 & 1 & 0 & 7 & 0 & 0 & 0 & 0 & 8 \\
\hline 6 & 0 & 0 & 2 & 0 & 0 & 0 & 0 & 2 \\
\hline 12 & 0 & 0 & 1 & 0 & 0 & 0 & 0 & 1 \\
\hline 13 & 0 & 0 & 1 & 0 & 0 & 0 & 0 & 1 \\
\hline 14 & 0 & 0 & 1 & 0 & 0 & 0 & 0 & 1 \\
\hline 18 & 0 & 0 & 1 & 0 & 0 & 0 & 0 & 1 \\
\hline 20 & 0 & 0 & 1 & 0 & 0 & 0 & 0 & 1 \\
\hline Total & 51 & 47 & 54 & 26 & 26 & 20 & 122 & 346 \\
\hline
\end{tabular}

Tabla 3. Cruce de la centralidad de autores por relación de colaboración entre especialidades científicas.

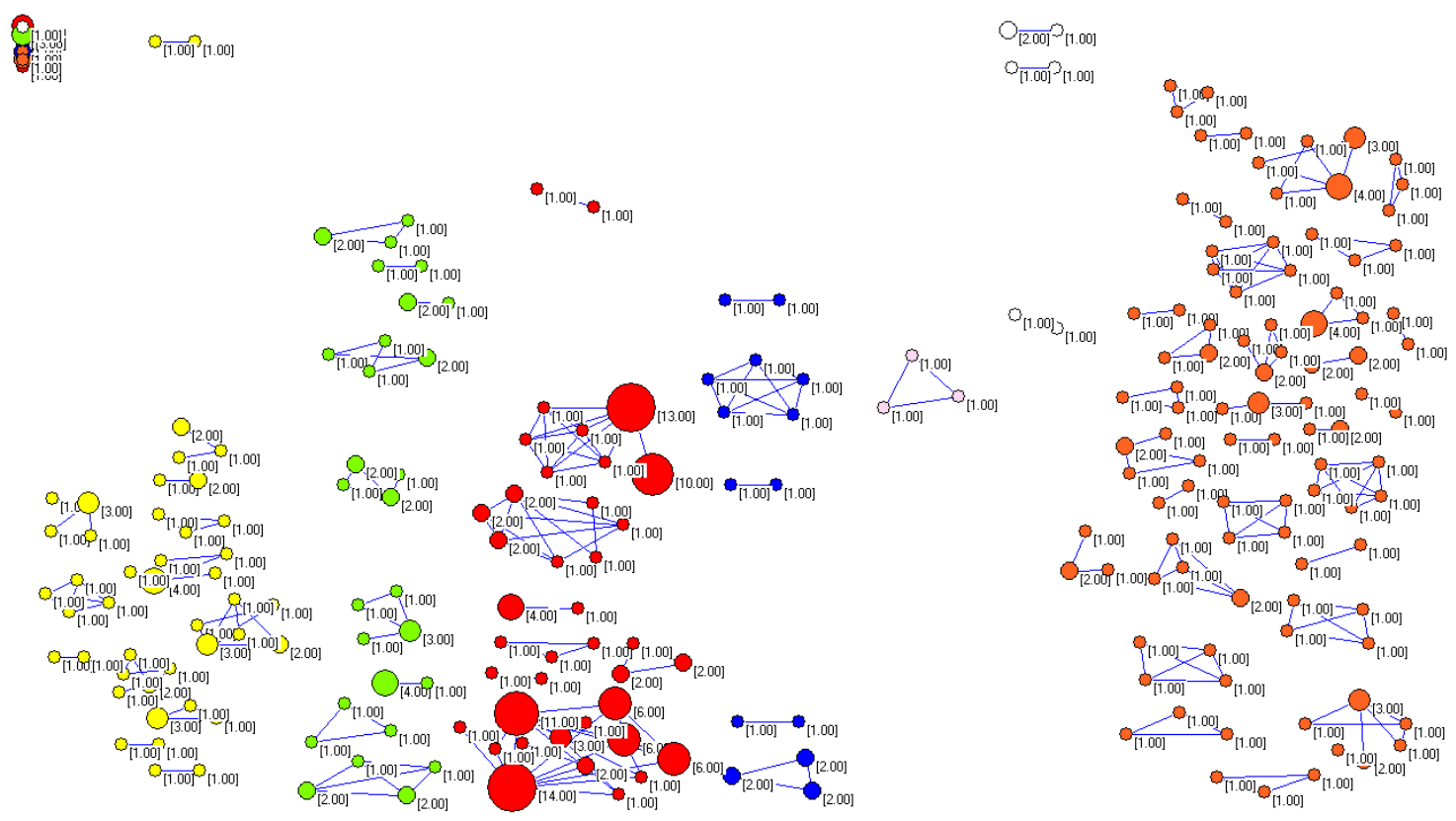

Figura 2. Productividad y centralidad por especialidades científicas.

Amarillo: biomedicina; verde: física; rojo: química; azul: biología; rosa: geociencias; blanco: ingeniería civil y metalurgia; y naranja: medicina general. El mayor tamaño de los nodos denota mayor productividad de un autor por año y los números al lado de cada punto se refieren a la cantidad de colaboraciones (centralidad) entre autores. Las relaciones o líneas son de co-autoría. 
En la figura 2 se puede corroborar la alta producción de la química en contraste por ejemplo con la escasa publicación de las ingenierías. Es notable la estructura colaborativa de la medicina general, sin embargo, también es visible su fragmentación en contraste con el campo de la química. De igual manera se nota en una exploración visual, lo parecido de las estructuras de la biomedicina y la medicina general. Se destaca el campo de la física por la aparición de componentes de más de dos autores a diferencia de la biología en donde son escasas las colaboraciones y con bajos niveles de centralidad.

\section{Núcleos de colaboración}

Otra medida de cohesión de la red y subredes de colaboración son los k-núcleos. Estos se refieren a la aparición de subredes las cuales se caracterizan por estar compuestas por autores que tienen determinado número de relaciones de colaboración y se relacionan con otros que tienen la misma cantidad o más relaciones de colaboración. Este tipo de medidas es relevante en tanto da cuenta de ciertas élites o grupos más visibles que recurren más a la colaboración y colaboran con otros que tienen un nivel parecido al suyo en cuanto a la realización de productos científicos en cooperación.

En la tabla 4 se cruzan especialidades científicas (columnas) y núcleos (filas) y se utliza el mismo procedimiento de la tabla 3. Cada número de cada núcleo corresponde al número de relaciones que tiene un autor con otros. Los números del interior de la tabla, corresponden al número de autores que se relacionan con otros autores de una disciplina y que tienen a su vez el mismo número de relaciones o corresponden al mismo núcleo.

\begin{tabular}{|c|c|c|c|c|c|c|c|c|}
\hline Relaciones & Biomedicina & Física & Química & Biología & Geociencias & Ingenierías & Medicina & Total \\
\hline 0 & 8 & 19 & 15 & 12 & 23 & 14 & 27 & 118 \\
\hline 1 & 20 & 8 & 9 & 6 & 0 & 6 & 30 & 79 \\
\hline 2 & 11 & 12 & 13 & 3 & 3 & 0 & 39 & 81 \\
\hline 3 & 12 & 8 & 1 & 0 & 0 & 0 & 16 & 37 \\
\hline 4 & 0 & 0 & 10 & 5 & 0 & 0 & 10 & 25 \\
\hline 5 & 0 & 0 & 6 & 0 & 0 & 0 & 0 & 6 \\
\hline Total & 51 & 47 & 54 & 26 & 26 & 20 & 122 & 346 \\
\hline
\end{tabular}

Tabla 4. Núcleos de colaboración por especialidad científica. 
La única especialidad científica en la que hay una subred en la que un autor tiene 5 o más relaciones de colaboración con otros autores que tienen la misma cantidad de relaciones de colaboración o más, es química. Esto implica la emergencia de nuevas formas de colaboración en la producción donde la cantidad de autores por obra científica es mayor. A pesar de que el campo científico de la química tiene una comunidad menor a la mitad de los miembros de la comunidad de medicina general, su emergencia como campo colaborativo es bastante relevante en la primera mitad del siglo XX. Por el contrario, la medicina general, a pesar de su tamaño tiene una cantidad menor de relaciones de colaboración entre autores con una alta relacionalidad colaborativa.

En colaboración, siguen siendo casi irrelevantes la biología, las geociencias y la ingeniería civil y metalurgia. Biomedicina y física tienen una estructura similar en su conformación de núcleos y es relevante su producción colaborativa, tomando en cuenta que supera en mucho los núcleos colaborativos a los puntos aislados.

\section{Colaboración en el tiempo: primera parte del siglo XX}

Como lo enuncia Roland Wagner (2001) la colaboración científica inició su expansión en forma significativa desde la Segunda Guerra Mundial, incluyendo en menor medida, a los países en desarrollo, entre ellos México, tal y como se muestra en el figura 3 y en la tabla 5.

\begin{tabular}{|c|c|c|c|c|c|c|}
\hline \multirow{2}{*}{ Colaboraciones } & \multicolumn{7}{|c|}{ Periodos } \\
\cline { 2 - 7 } & $\begin{array}{c}\mathbf{1 9 0 0 -} \\
\mathbf{1 9 1 0}\end{array}$ & $\begin{array}{c}\mathbf{1 9 1 1 -} \\
\mathbf{1 9 2 0}\end{array}$ & $\begin{array}{c}\mathbf{1 9 2 1 -} \\
\mathbf{1 9 3 0}\end{array}$ & $\begin{array}{c}\mathbf{1 9 3 1 -} \\
\mathbf{1 9 4 0}\end{array}$ & $\begin{array}{c}\mathbf{1 9 4 1 -} \\
\mathbf{1 9 5 0}\end{array}$ & Total \\
\hline 0 & 7 & 9 & 19 & 28 & 55 & 118 \\
\hline 1 & 2 & 4 & 0 & 8 & 59 & 73 \\
\hline 2 & 0 & 0 & 0 & 16 & 55 & 71 \\
\hline 3 & 0 & 0 & 0 & 0 & 48 & 48 \\
\hline 4 & 0 & 0 & 0 & 6 & 15 & 21 \\
\hline 5 & 0 & 0 & 0 & 0 & 8 & 8 \\
\hline 6 & 0 & 0 & 0 & 0 & 2 & 2 \\
\hline
\end{tabular}

Tabla 5. Cantidad de colaboraciones por autor en un año, distribuidos por décadas.

En la tabla 5, las columnas corresponden a las 5 primeras décadas del siglo XX, las filas corresponden a la cantidad de artículos hechos en colaboración (el cero representa autorías únicas). Los números de la tabla representan la cantidad de colaboraciones por año. La tabla igualmente ha sido elaborada con el software Pajek (De Nooy W, Mrvar A, Batagelj, V, 2005) 
Se pueden observar algunas primeras colaboraciones con pocas relaciones en las dos primeras décadas. La tercera década es nula en cuanto a colaboración (tal vez una de las razones, es el período de guerra revolucionario) y una explosión que comienza paulatinamente en la década de los años 30 y se consolida en los 40, tal y como ocurre en los centros de la ciencia en el mundo (Europa y Estados Unidos para la época). Pero no es sólo relevante la aparición de multiplicidad de formas colaborativas en los distintos campos científicos, sino también la quintuplicación en la aparición de 58 autores en la década de los 30, a 247 en la década de los 40 .

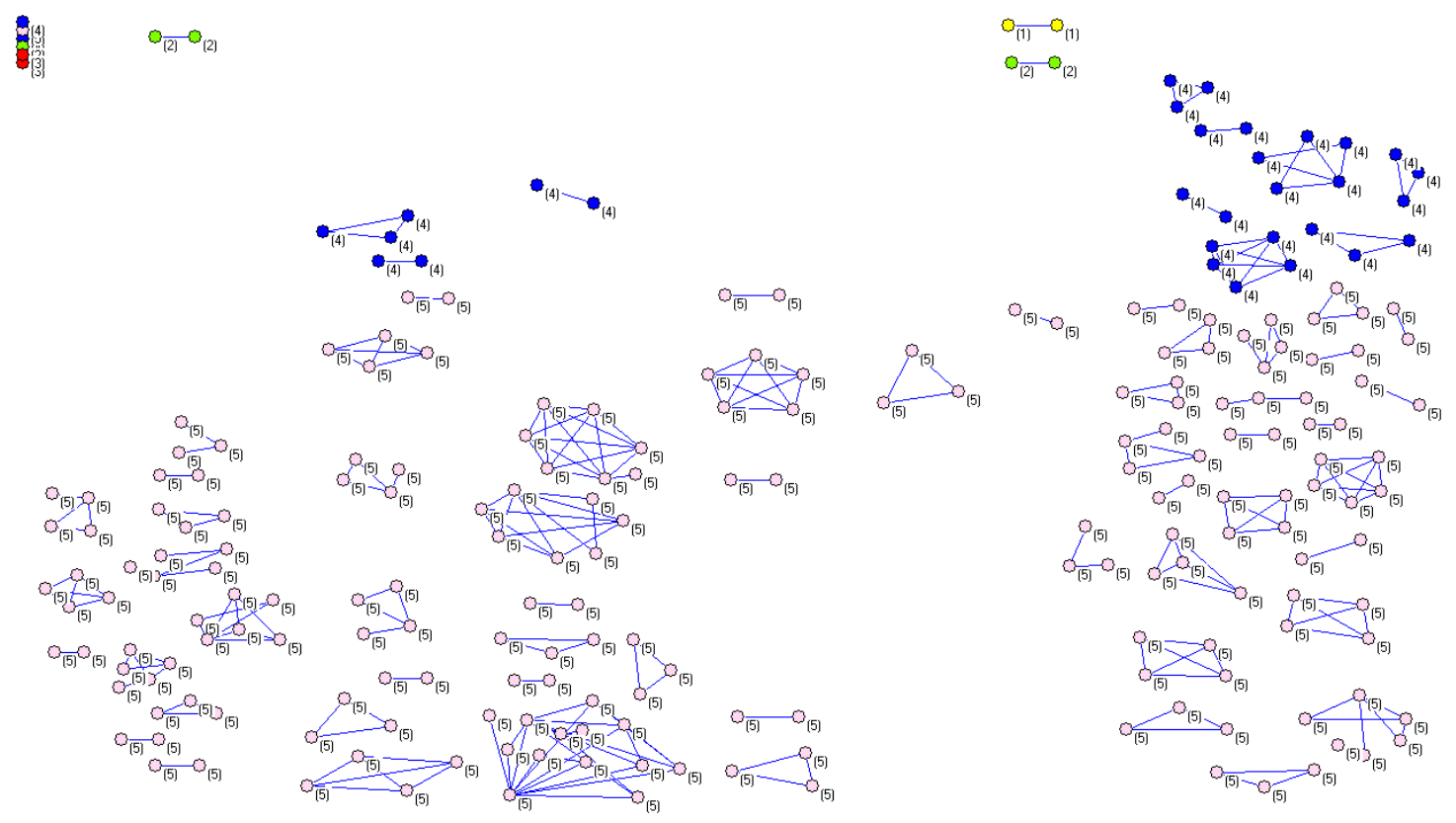

Figura 3. Relaciones de colaboración en el tiempo: décadas 1900 a 1950.

Amarillo: 1900-1910; verde: 1911-1920; rojo: 1921-1930; azul: 1931-1940; rosa: 1941-1950. Los puntos al extremo izquierdo corresponden a autores que producen aisladamente. Cada nodo corresponde a un autor, cada línea a una o más relaciones de co-autoría.

En la misma distribución temporal se presenta la figura 4 por disciplinas de izquierda a derecha. Si se contrastan las figuras 2 y 3 , se puede observar la explosión colaborativa. Excepto la biología, las geociencias y la ingeniería civil y metalurgia, el resto de las especialidades consolidaron una estructura colaborativa generando mayores procesos de institucionalización y continuidad en los programas de investigación a través de la expansión posible en las colaboraciones, no sólo entre científicos, sino también interinstitucionales.

Sólo tomando en cuenta la producción en la década de los años 40 se encuentran únicamente 57 producciones aisladas de 247 autores en relación. En comparación 
con las décadas anteriores en donde en los años 30 se encuentra 58 autores en los años 20, 19; en la década del 10, 13 y los primeros años del siglo con 9 autores.

Se puede destacar que el primer antecedente de colaboración detectado se encuentra en la primera década del siglo $X X$, sin muchos progresos hasta la década de los años 30, consolidándose la cooperación en la década de los 40.

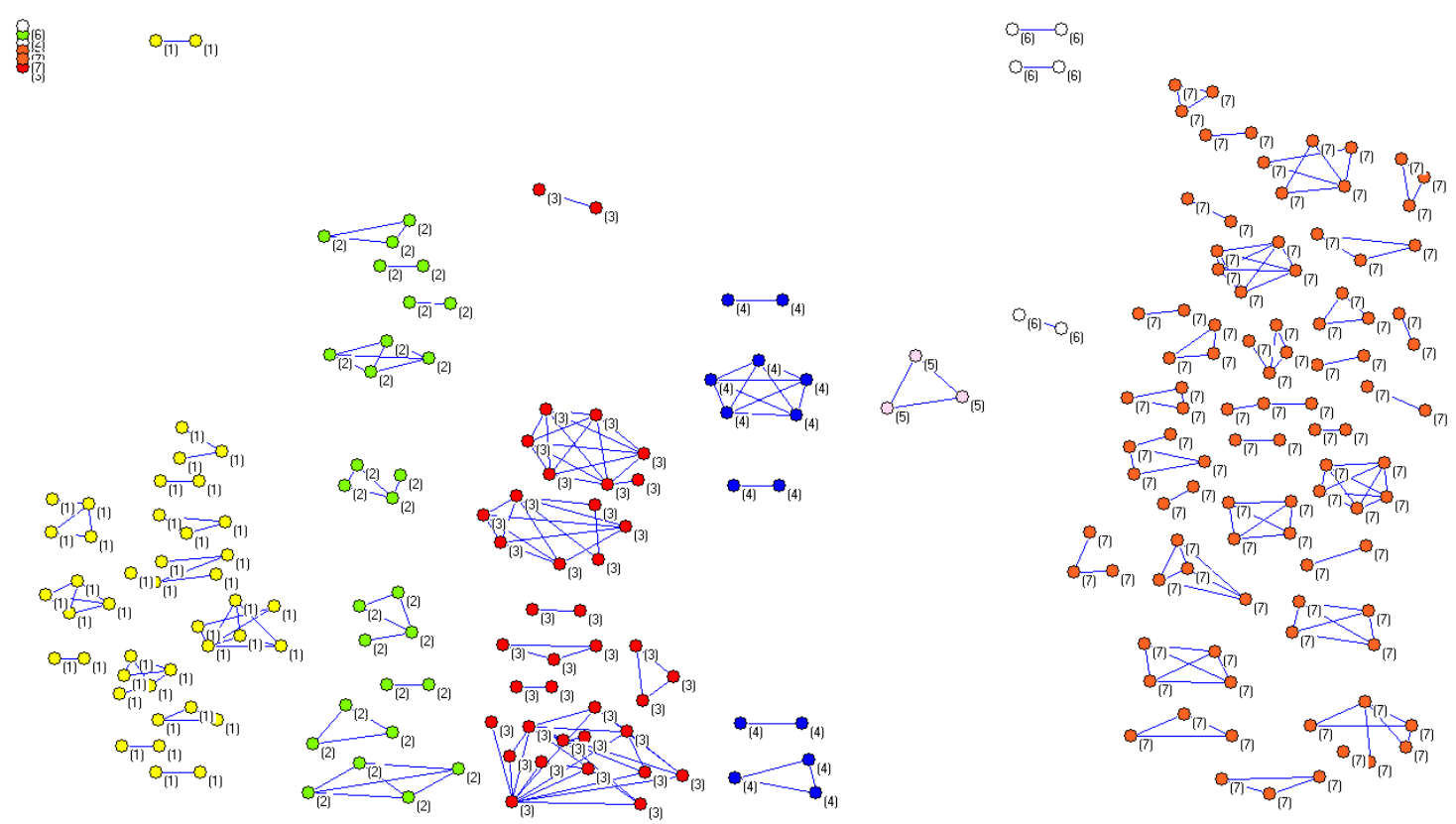

Figura 4. Relaciones de colaboración en el tiempo por disciplinas.

Amarillo (biomedicina); verde (física); rojo (química); azul (biología); rosa (geociencias); blanco (ingeniería civil y metalurgia); naranja (medicina general). Esta figura presenta la misma distribución de la anterior, excepto que cambian los atributos de los autores. En la figura anterior están distribuidos por décadas, en ésta por especialidad científica.

\section{Conclusiones}

Es importante recordar que las conclusiones sólo se aplican a la producción científica de corriente principal disponible en las bases de datos Web of Science y SCOPUS. Si bien no describen la totalidad de la producción científica, si la más relevante en las clasificaciones internacionales y con mayor visibilidad. Por tanto las conclusiones, si bien no se aplican para toda la ciencia mexicana, por lo menos si son aplicables para la parte más visible y por tanto la que mayor posibilidad tiene de ser discutida en las comunidades académicas internacionales. 
En este sentido, la ciencia tiene sus propias dinámicas de construcción y creación: creciente especialización y subespecialización. En México, si bien es cierto que responde a la misma lógica constructiva global de la ciencia, ésta cobra dinamismo por diseminación y expansión de sus dominios a través de la academia y la socialización de algunos científicos con la ciencia europea y estadounidense. Hay unas características especiales del entorno geográfico en donde se conjugaron otras formas de racionalidad que afectaron el desempeño mismo de esta como lo muestran los resultados. Esto puede observarse en la coincidencia de algunos hechos históricos con el aumento, la disminución o la escasa aparición de la colaboración en la producción científica.

Se demostró que el establecimiento de relaciones de coautoría como práctica común, actúa como elemento de acumulación de capital científico. Esto se puede observar en el análisis exploratorio de las redes de co-autoría en el que las áreas como la biomedicina, la medicina y la química muestran su prominencia colaborativa. La física seguiría en el orden de colaboración, pero relevante en tanto su pequeño campo en comparación con el de la medicina, logra realizar casi la totalidad de su trabajo en colaboración, lo que implica cierto grado de consolidación de un campo naciente en México.

Desde un punto de vista historiográfico, el proceso de transición de las prácticas científicas aisladas a las formas de organización colaborativas, ocurrido en la mayoría de las disciplinas de la ciencia mexicana de la primera mitad del siglo XX, lleva cierta impronta del contexto cultural (Burton, 1991) del periodo nacionalista posrevolucionario (Castañeda y Zamudio, 1989), desarrollado entre las décadas de 1930 a 1950. De esta forma la preocupación por el desarrollo social en la erradicación de enfermedades epidémicas impulsa los esfuerzos colaborativos en la medicina y la biomedicina. El desarrollo de la economía global en la posguerra, también toca a México en el área de la química, dando un fuerte impulso a la institucionalización de este campo en aquella época. Por otro lado, curiosamente la nacionalización de los recursos naturales por parte del Estado, no afecta de manera positiva la producción científica en las áreas de ingenierías y metalurgia necesarias para la explotación de recursos como el petróleo por ejemplo o para el desarrollo de maquinarias para la posible industria agropecuaria, lo cual tal vez exprese un desinterés por el desarrollo tecnológico a través del conocimiento, llevando a posibles dependencias de tecnologías extranjeras en ese entonces.

En la contrastación tanto de datos historiográficos como analíticos es posible dar cuenta, desde un punto de vista cualitativo, de una correlación entre los 
mecanismos de institucionalización de la ciencia y el crecimiento de la colaboración científica. De esta manera los campos de la medicina, biomedicina, física y química crecen en conformación de grupos colaborativos, en la medida en que crecen las condiciones institucionales de desarrollo de cada uno de estos campos especializados.

No se puede dejar de lado el papel que también juegan los individuos en la construcción de comunidades académicas. Así, en campos como la química, la física, la medicina y la biomedicina, es importante el papel de algunos investigadores con alta productividad que logran generar convocatoria de otros investigadores con menor productividad para generar trabajos colaborativos. Las otras disciplinas que cuentan con autores que tienen bajos niveles de productividad, tienen un menor desarrollo de trabajos colaborativos y coincide con menores condiciones institucionales históricas. De esta manera la correlación podría entenderse no como causal sino de mutua determinación entre condiciones históricas y capacidades personales de los investigadores.

Finalmente, visualizando un programa de investigación a futuro, este estudio exploratorio de las relaciones de colaboración científica en la producción y difusión, arrojan varias líneas de trabajo posibles:

Primero. La explosión de la colaboración científica parece estar muy ligada a la institucionalización y a un desarrollo organizativo propicio para su desarrollo. Los campos más colaborativos coinciden con la atención dada a la investigación por otros sistemas. Esto lleva a una interesante línea de trabajo futuro acerca de las vinculaciones de la ciencia mexicana con los distintos sistemas sociales: económico, político y educativo.

Segundo. En México, al igual que en los países europeos y Estados Unidos, durante y después de la segunda guerra mundial se empezaron a generar nuevas formas de organización en la ciencia, considerando como una de sus consecuencias, la proliferación de la colaboración científica. No se puede concluir de ello que la ciencia tuviese un desarrollo igual que en otros países. Lo interesante en realidad son los factores que han permitido o no este tipo de vinculaciones entre la ciencia y la sociedad.

Tercero. Este tipo de hallazgos realizados son relevantes también para el campo de la bibliometría, en tanto que expanden sus posibilidades. La producción científica estudiada por la bibliometría, si se observa como la materia prima de la descripción de distintos campos científicos y sus adscripciones institucionales, en un análisis amplio, incluyendo sus interrelaciones con otros sistemas sociales, podría aportar 
entendimiento de las formas de vinculación entre la ciencia misma y otros sistemas sociales. También sería importante, en cierto sentido, observar como estos acoplamientos, de alguna u otra manera van moldeando las formas de organización científicas. 


\section{Bibliografía}

Burton, C. (1991). El sistema de educación superior: una visión comparativa de la organización académica. México: Nueva Imagen: Universidad Futura: UAM, 54 p.

Castañeda-Castillo, R.M. y Zamudio-Varela, G. (1989). El nacionalismo en la historia de la ciencia mexicana de mediados del siglo XX. En Memorias del Primer Congreso Mexicano de Historia de la Ciencia y de la Tecnología. México: Sociedad Mexicana de la Historia de la Ciencia y la Tecnología. Tomo 1: 166-171.

Collazo-Reyes, F. y Herrera-Corral, G. (2008). Alfredo Baños y el surgimiento de la física y la investigación académica en México. Avance y Perspectiva, 1(1): 5-19

De Gortari, E (1963). La ciencia en la historia de México. México; FCE, 1963.

De Nooy, W., Mrvar, A. and Batagelj, V. (2005). Exploratory Social Network Analysis with Pajek. Nueva York: Cambridge University Press, 320 p.

Delgado-López, E., Torres-Salinas, D., Jiménez-Contreras, E. y Ruíz-Pérez, R. (2006). Análisis bibliométrico y de redes sociales aplicado a las tesis bibliométricas defendidas en España (1976-2002): temas, escuelas científicas y redes académicas. Revista Española de Documentación Científica, 29 (4): 1-36.

Ledesma-Mateos, I, y Barahona-Echeverría, A. (1999). Alfonso Luis Herrera E Isaac Ochotorena: la institucionalización de la biología en México. México: UNAM, 40 p. Disponible en:

http://historiamexicana.colmex.mx/pdf/13/art_13_1873_16030.pdf, (Enero, 2008).

Leydesdorff, L. y Olga, A. (1990). Dimensions of Citation Analysis. Science, Technology, \& Human Values, 15 (3): 305-335.

Leydesdorff, L. (2001). A Sociological Theory of Communication. UPublishers: Boca Ratón (Florida).

Leydesdorff, L. (2008). International collaboration in science and the formation of a core group. Journal of Informetrics, 2: 317-325

Luhmann, N. (1996). La ciencia de la sociedad. México: UIA, 516 p.

Luhmann, N. (2007). La Sociedad de la Sociedad. México: Herder, 590 p.

Luna-Morales, M.E., Collazo-Reyes, F. and Russell, J. (2007). A quantitative Historiography of Mexican Integration into the International standards of Scientific Research. En Proceedings of International Conference of the International Society for Scientometrics and Informetrics, Madrid, Spain, June 25-27. pp. 523-530. 
Martínez, S. (2003). Geografía de las prácticas científicas. Racionalidad, heurística y normatividad. México; UNAM, 206, p.

Pontille, D. (2004). Scientific authorship. Credit and intellectual property in science. Revue Francaise de Sociologie, 45(2): 374-377.

Pontille, D. (2003a). Authorship practices and institutional contexts in sociology: Elements for a comparison of the United States and France. SCIENCE TECHNOLOGY $\&$ HUMAN VALUES, 28(2): 217-243.

Pontille, D, (2003b). Format's D'Ecriture Et Mondes Scientifiques: Le Cas de la Sociologie. Questions de Communication, 3: 55-67.

Pérez Tamayo, R (2005) Historia general de la ciencia en México. México: FCE, 319 p. ISBN 968-16-7861-3

Robles-Glenn, J. (1971). La investigación mexicana y los índices extranjeros de información. Anuario de Bibliotecología, Archivología e Informática, 3(2): 48-99

Saldaña, J.J. y Cuevas Cardona, C. (2005). La invención en México de la investigación científica profesional: el Museo Nacional (1868-1908). En La Casa de Salomón en México / Coordinador Juan J osé Saldaña. México; UNAM, 185-216 p.

Saldaña, J.J. y Azuela, L.F. (1994). De amateurs a profesionales. Las sociedades científicas en México en el siglo XIX. Quipu, 11(2), 135-172.

Suárez, E. (2005). La historiografía de la ciencia. En historia, filosofía y enseñanza de la ciencia, S.F. Martínez, \& G. Guillaumin (comps.). México: UNAM. Instituto de Investigaciones Filosóficas, pp. 17-42.

Valiente-Banuet, A., Flores-Hernández, N., Verdú, M. y Dávila, P. (1998). The chaparral vegetation in México under nonmediterranean climate: the convergence and Madrean Tethyan hypotheses reconsidered. American Journal of Botany, 85: 1398- 1408.

Velden, T., Haque, A., Lagoze, C.A. (2010). New approach to analyzing patterns of collaboration in co-authorship networks: mesoscopic analysis and interpretation. Scientometrics, 85(1): 219-242.

Wagner-Döbler, R. (2001). Continuity and discontinuity of collaboration behaviour since 1800 - from a bibliometric point of view. Scientometrics, 52 (3): 503-517. 
Yoshikane F., Nozawa T., Shibui S... et al. (2009) An analysis of the connection between researchers' productivity and their co-authors' past attributions, including the importance in collaboration networks. Scientometrics, 79(2): 435-449

Ziman, J. (1994). Prometheus bound: science in a dynamic steady state. Cambridge: University Press, 289 p. 


\section{ANEXOS}

\begin{tabular}{|c|c|c|}
\hline Sectores & Subsectores & Dependencias, año de creación y áreas de interés \\
\hline \multirow{6}{*}{ Académico } & UNAM & $\begin{array}{l}\text { Antropológicas (1939); Arquitectura (1935); Artes Plásticas (1935); } \\
\text { Económicas (1940); Estéticas (1935); Estudios Médicos y Biológicos } \\
\text { (1941); Facultad Ciencias (1930); Física (1938); Geofísica (1945); } \\
\text { Geografía (1938); Geográficas (1933); Históricas (1945); Medicina y } \\
\text { Veterinaria (1945); Química (1941); Matemáticas (1942) ; Sociales } \\
\text { (1930) }\end{array}$ \\
\hline & $\begin{array}{l}\text { Institutos } \\
\text { Tecnológicos }\end{array}$ & $\begin{array}{l}\text { Biotécnico (1934); Tecnológico de Durango (1948); Investigaciones } \\
\text { Agrícolas (1947); Científico y Literario de Toluca (1943) }\end{array}$ \\
\hline & IPN & $\begin{array}{l}\text { Escuelas: Bacteriología, Parasitología y Fermentaciones (1937); } \\
\text { Nacional de Ciencias Biológicas (1936); Superior de Medicina (1938); } \\
\text { Superior de Ingeniería Química e Industrias Extractivas (1947), Esc. } \\
\text { Sup. Ingeniería Mecánica y Eléctrica (1932) }\end{array}$ \\
\hline & Univ. Estatales & $\begin{array}{l}\text { Guadalajara (1935); Nuevo León (1943); Colima (1940); Puebla (1937); } \\
\text { Sinaloa (1941); Socialista Noreste (1937); Sonora (1942); Veracruz } \\
\text { (1944); Yucatán (1936) }\end{array}$ \\
\hline & Privado & $\begin{array}{l}\text { Instituto Tecnológico Autónomo de México (1946); Instituto Tecnológico } \\
\text { de Estudios Superiores de Monterrey (1943) }\end{array}$ \\
\hline & Investigación & $\begin{array}{l}\text { Casa de España (1938); Colegio México (1940); Esc. Textil Río Blanco } \\
\text { (1933); Escuela Superior de Ingeniería Mecánica y Eléctrica (1932): } \\
\text { Observatorio Astrofísico Nacional de Tonantzintla (1942) }\end{array}$ \\
\hline Gobierno & Dependencias & $\begin{array}{l}\text { Departamentos: Autónomo Forestal de Caza y Pesca (1934); Marina } \\
\text { Nacional (1939); Direcciones: Geografía y Meteorología (1935); } \\
\text { Geografía, Meteorología e Hidrología (1935); Secretarías: Agricultura y } \\
\text { Ganadería (1946); Defensa Nacional (1937); Marina (1941); Servicio } \\
\text { Geológico Mexicano (1944); Instituto Nacional de Bellas Artes (1946); } \\
\text { Investigaciones Forestales (1932); Oficina Federal para la Defensa } \\
\text { Agrícola (1933); Instituto Mexicano de Investigaciones Tecnológicas } \\
\text { (1946); }\end{array}$ \\
\hline \multirow[t]{2}{*}{ Industrial } & $\begin{array}{l}\text { Química } \\
\text { Farmacéutica }\end{array}$ & $\begin{array}{l}\text { Armour Research Foundation (1949); Camex, S.A (1945); Celanese } \\
\text { Mexicana (1947); Grupo Cydsa (1945); Laboratoire d' Analyses } \\
\text { Chimiques (1930); Laboratorio Syntex, S.A (1944); Laboratorios de } \\
\text { Fomento Industrial (1948); } \\
\text { Laboratorio Hormona S.A. (1933); Sosa Texcoco (1942) }\end{array}$ \\
\hline & $\begin{array}{l}\text { Minero } \\
\text { Metalúrgica }\end{array}$ & $\begin{array}{l}\text { American Mer Co LTD (1939); Beneficiadora de Pachuca (1930); Tigris } \\
42 \text { (1947) }\end{array}$ \\
\hline \multirow[t]{4}{*}{ Salud } & $\begin{array}{l}\text { Secretaría de } \\
\text { Salubridad y } \\
\text { Asistencia }\end{array}$ & $\begin{array}{l}\text { Hospitales: General México (1939); Infantil México (1943); Institutos: } \\
\text { Cancerología (1946); Cardiología (1944); Manual Gea González (1947); } \\
\text { Ministerio de Salud Publica y Bienestar (1946); Neuropsiquiatría (1943); } \\
\text { Nutrición (1946); Salubridad y Enfermedades Tropicales (1939) }\end{array}$ \\
\hline & IMSS & Instituto Mexicano del Seguro Social (1943) \\
\hline & Privada & $\begin{array}{l}\text { Centro Dermatológico Pascua (1937); Centro Médico de } \\
\text { Chihuahua (1949); Sanatorio Tuberculosos de Huipulco (1936); } \\
\text { Clínica Londres (1931) }\end{array}$ \\
\hline & Otras & $\begin{array}{l}\text { Hospital Central Militar (1942); Laboratorios de Investigación: } \\
\text { (1937); Brucelosis (1939); Plasmogenia (1939) }\end{array}$ \\
\hline
\end{tabular}

Tabla 6. Instituciones y dependencias relacionadas con actividades de investigación creadas en el Periodo 1930-1950. 


\begin{tabular}{|c|c|c|}
\hline Sectores & Subsectores & Dependencias, año de creación y áreas de interés \\
\hline \multirow{4}{*}{$\begin{array}{l}\text { Asociaciones } \\
\text { Científicas }\end{array}$} & Medicina & $\begin{array}{l}\text { Academias Mexicanas de: Cirugía (1933); de Medicina Veterinaria } \\
\text { (1933); Asociaciones mexicanas de: Cardiología (1935); Dermatología } \\
\text { (1936); Ginecología y Obstetricia (1947); Higiene (1944); Médica de } \\
\text { Guadalajara (1938); Oftalmología y Otorrinolaringología ( 1943); } \\
\text { Neumología y Cirugía de Tórax (1939); Neurología y Psiquiatría } \\
\text { (1937); Ortopedia (1946); Otorrinolaringología y Bronco-Esofagología } \\
\text { (1946); Pediatría (1930); Radiología y Fisioterapia (1942); Cirujanos } \\
\text { Dentistas (1942); Gastroenterología (1935) }\end{array}$ \\
\hline & Humanidades & Sociedad Mexicana de Antropología (1937) \\
\hline & Ciencias & $\begin{array}{l}\text { Sociedad mexicana de: Ciencias Física (1943); Matemáticas (1943); } \\
\text { Botánica de México (1941) }\end{array}$ \\
\hline & Otras & Academia Mexicana de Genealogía y Heráldica (1943) \\
\hline Instituciones & $\begin{array}{l}\text { De promoción a } \\
\text { la ciencia }\end{array}$ & $\begin{array}{l}\text { Academia Nacional de Ciencias (1933); Consejo Nacional de la } \\
\text { Educación Superior y de Investigación Científica (1935); El Colegio } \\
\text { Nacional (1940); Comisión Impulsora y Coordinadora de la } \\
\text { Investigación Científica en México (1943); Colegio de Ingenieros } \\
\text { Mecánicos y Electricistas A.C. (1945) }\end{array}$ \\
\hline
\end{tabular}

Tabla 7. Asociaciones civiles y entidades gubernamentales de promoción y financiamiento de la ciencia: 1930-1950 